East Tennessee State University

Digital Commons@ East Tennessee State University

ETSU Faculty Works

Faculty Works

$12-10-2018$

\title{
On the Apparent Absence of Wolf-Rayet+Neutron Star Systems: The Curious Case of WR124
}

Jesus A. Toala

UNAM Campus Morelia

Lidi Oskinova

University of Potsdam

W.R. Hamann

University of Potsdam

Richard Ignace

East Tennessee State University, ignace@etsu.edu

A.A. C. Sander

University of Potsdam

See next page for additional authors

Follow this and additional works at: https://dc.etsu.edu/etsu-works

\section{Citation Information}

Toala, Jesus A.; Oskinova, Lidi; Hamann, W.R.; Ignace, Richard; Sander, A.A. C.; Todt, H.; Chu, Y.H.; Guerrero, M. A.; Hainich, R.; Hainich, R.; and Terrejon, J. M.. 2018. On the Apparent Absence of Wolf-Rayet+Neutron Star Systems: The Curious Case of WR124. Astrophysical Journal Letters. Vol.869 https:// doi.org/10.3847/2041-8213/aaf39d ISSN: 2041-8205 accepted for inclusion in ETSU Faculty Works by an authorized administrator of Digital Commons @ East Tennessee State University. For more information, please contact digilib@etsu.edu. 


\section{On the Apparent Absence of Wolf-Rayet+Neutron Star Systems: The Curious Case of WR124}

\section{Copyright Statement}

(C) 2018. The American Astronomical Society. Reproduced by permission of the AAS.

\section{Creator(s)}

Jesus A. Toala, Lidi Oskinova, W.R. Hamann, Richard Ignace, A.A. C. Sander, H. Todt, Y.H. Chu, M. A. Guerrero, R. Hainich, R. Hainich, and J. M. Terrejon 


\title{
On the Apparent Absence of Wolf-Rayet+Neutron Star Systems: The Curious Case of WR124
}

\author{
J. A. Toalá (杜宇君) $)^{1}$ (D) L. M. Oskinova ${ }^{2,3}$, W.-R. Hamann ${ }^{2}$, R. Ignace ${ }^{4}$ (1) A. A. C. Sander ${ }^{2,5}$, T. Shenar ${ }^{2}$ (1), \\ H. Todt ${ }^{2}$, Y.-H. Chu (朱有花) $)^{6}$, M. A. Guerrero ${ }^{7}$ (10), R. Hainich ${ }^{2}$, and J. M. Torrejón ${ }^{8}$ (1) \\ ${ }^{1}$ Instituto de Radioastronomía y Astrofísica, UNAM Campus Morelia, Apartado Postal 3-72, Morelia 58090, Michoacán, Mexico; j.toala@irya.unam.mx \\ ${ }^{2}$ Institute for Physics and Astronomy, University of Potsdam, D-14476 Potsdam, Germany; lida@astro.physik.uni-potsdam.de \\ ${ }^{3}$ Kazan Federal University, Kremlevskaya Str 18, 420008, Kazan, Russia \\ ${ }^{4}$ Department of Physics and Astronomy, East Tennesse State University, Johnson City, TN 37611, USA \\ ${ }^{5}$ Armagh Observatory and Planetarium, College Hill, Armagh BT61 9DG, UK \\ ${ }^{6}$ Institute of Astronomy and Astrophysics, Academia Sinica (ASIAA), Taipei 10617, Taiwan \\ ${ }^{7}$ Instituto de Astronomía de Andalucía, IAA-CSIC, Glorieta de la Astronomía S/N, Granada E-18008, Spain \\ ${ }^{8}$ Instituto Universitario de Física Aplicada a las Ciencias y las Tecnologías, Universiad de Alicante, Alicante E-0380, Spain \\ Received 2018 August 8; revised 2018 October 29; accepted 2018 November 19; published 2018 December 6
}

\begin{abstract}
Among the different types of massive stars in advanced evolutionary stages is the enigmatic WN8h type. There are only a few Wolf-Rayet (WR) stars with this spectral type in our Galaxy. It has long been suggested that WN8htype stars are the products of binary evolution that may harbor neutron stars (NS). One of the most intriguing WN8h stars is the runaway WR 124 surrounded by its magnificent nebula M1-67. We test the presence of an accreting NS companion in WR 124 using $\sim 100$ ks long observations by the Chandra X-ray observatory. The hard X-ray emission from WR 124 with a luminosity of $L_{\mathrm{X}} \sim 10^{31} \mathrm{erg} \mathrm{s}^{-1}$ is marginally detected. We use the non-local thermodynamic equilibrium stellar atmosphere code PoWR to estimate the WR wind opacity to the X-rays. The wind of a WN8-type star is effectively opaque for X-rays, hence the low X-ray luminosity of WR 124 does not rule out the presence of an embedded compact object. We suggest that, in general, high-opacity WR winds could prevent X-ray detections of embedded NS, and be an explanation for the apparent lack of WR+NS systems.
\end{abstract}

Key words: circumstellar matter - ISM: jets and outflows - stars: massive - stars: evolution - stars: neutron stars: Wolf-Rayet

\section{Introduction}

The evolutionary paths of stars more massive than $\mathrm{M}_{\mathrm{i}} \gtrsim 40 M_{\odot}$ are not well established. It is accepted that when these stars evolve off the main sequence their mass-loss rates increase significantly, and they reach the Wolf-Rayet (WR) phase before ending their lives in a core-collapse event. Some of these objects may pass through a short $\left(\sim 10^{5}\right.$ yr) luminous blue variable (LBV) evolutionary stage that is characterized by high mass-loss rates and outbursts (e.g., Jiang et al. 2018). The ejected material, up to several $M_{\odot}$, is often observed in the form of associated nebulae. WR stars that display CNO-processed matter in a strong stellar wind are classified as part of the nitrogen sequence (WN type). The cooler, late WN subtypes (WNL) usually contain some hydrogen in their atmospheres, while the hotter, early subtypes (WNE) are usually hydrogen free (Hamann et al. 2006). WNL stars are often embedded in remnant LBV nebulae (e.g., Barniske et al. 2008). On the other hand, WNE stars with their faster stellar winds are often surrounded by ring nebulae filled by shocked X-ray emitting plasma (see Toalá et al. 2017, and references therein). The WN phase may be followed by the WC/WO stage, when the products of helium burning appear in the stellar atmosphere (Sander et al. 2018).

A significant fraction of WR stars are either a direct product of massive binary evolution or experienced binary interactions during their lifetimes (e.g., Paczynski 1967; Shenar et al. 2016). Massive binary evolutionary models predict the formation of high-mass X-ray binaries (HMXBs) with a WR-type donor and a black hole (BH) or a neutron star (NS; e.g., Postnov \& Yungelson 2014). The compact object may accrete matter either as stellar wind or via Roche lobe overflow. van den Heuvel et al. (2017) demonstrated that WR+NS systems cannot be formed through stable mass transfer. It is therefore very likely that the formation of a WR $+\mathrm{NS}$ system takes place via a common envelope (CE). MacLeod \& Ramirez-Ruiz (2015) showed that BHs and NSs can efficiently gain mass during $\mathrm{CE}$ evolution but avoid runaway growth. Provided the system is initially wide enough, the ejection of the CE is expected (Terman et al. 1995). Ejected material might form a young circumstellar nebula.

Population synthesis studies predict a significant number of $\mathrm{WR}+\mathrm{NS} / \mathrm{BH}$ binaries. Vanbeveren et al. (1998) suggested that among WR stars, 3\%-5\% may have a $\mathrm{BH}$ companion and $2 \%-8 \%$ may have a NS companion. The latter originate from $\mathrm{OB}+\mathrm{NS}$ progenitors that survived previous spiral-in. De Donder et al. (1997) predicted at least three WR+NS systems should be present within $3 \mathrm{kpc}$ from the Sun, while the more recent population synthesis models predict that there should be $\sim 500$ WR+NS binaries in the Galaxy (Lommen et al. 2005).

These predictions are confronted with X-ray observations that find only a handful of WR stars with relativistic companions, typically BHs. Only one of them, Cyg X-3, is in the Galaxy, (van Kerkwijk et al. 1992). Being X-ray bright $\left(L_{\mathrm{X}} \approx 10^{38} \mathrm{erg} \mathrm{s}^{-1}\right)$, it most likely harbors a low-mass $\mathrm{BH}$ (e.g., Koljonen et al. 2018).

Different explanations seek to resolve the tensions between theoretical expectations and X-ray observations. Vanbeveren et al. (1998) considered the spinning of an NS during the spiralin, and suggested that high resulting spin may inhibit accretion. Accretion might also be suppressed by magnetic gating mechanisms (see Bozzo et al. 2016, and references therein). De Donder et al. (1997) predicted a population of weird WR stars, i.e., WR stars with NSs in their centers as a result of 
Table 1

Properties of WR 124

\begin{tabular}{lc}
\hline \hline Spectral type $^{1}$ & WN8h \\
Mass $[M]^{1}$ & $33 M_{\odot}$ \\
Luminosity $\left[\log _{10}(L)\right]^{1}$ & $6.0 L_{\odot}$ \\
Radius $\left[R_{\star}\right]$ & $16.7 R_{\odot}$ \\
Mass-loss rate $[\dot{M}]^{1}$ & $8 \times 10^{-5} M_{\odot} \mathrm{yr}^{-1}$ \\
Wind velocity $\left[v_{\infty}\right]^{1}$ & $710 \mathrm{~km} \mathrm{~s}^{-1}$ \\
ISM column density $\left.^{1} \log N_{\mathrm{H}} \mathrm{cm}^{-2}\right]$ & 21.5 \\
Distance $[d]^{2}$ & $8.7 \mathrm{kpc}$ \\
Stellar velocity $\left[v_{\star}\right]^{3}$ & $190 \mathrm{~km} \mathrm{~s}^{-1}$ \\
${\text { Variability period }[P]^{4}}^{4}$ & $\sim 2.4$ days $^{1}$ \\
\hline
\end{tabular}

Note. ${ }^{1}$ Hamann et al. (2006), ${ }^{2}$ Gaia Collaboration et al. (2018), ${ }^{3}$ Kharchenko et al. (2007), ${ }^{4}$ Moffat et al. (1982).

mergers. These stars are thought to be the cousins of ThorneŻytkow objects (TŻOs). Another explanation considers the possibility that a WR wind absorbs X-rays from an embedded accreting NS/BH (Vanbeveren et al. 1998; Lommen et al. 2005). Recent progress in understanding WR winds has indeed shown that some WR stars have very high opacity to X-rays (Oskinova et al. 2003).

Among the various types of WR stars, the WN8-type has long been considered peculiar as compared to other WR subtypes (Moffat 1989). These stars are variable, have low binary fraction, and often are runaways with high Galactic altitudes, suggesting that they were kicked away from the Galactic disk by a supernova (SN) explosion (De Donder et al. 1997; Marchenko et al. 1998b; Chené et al. 2011). These characteristics led to speculations that WN8-type stars might be TŻOs (Foellmi \& Moffat 2002).

In this Letter we present Chandra X-ray observations of WR 124, arguably the most probable WR+NS candidate in our Galaxy. Our observations are used to discuss the possible presence of a NS embedded in the wind of WR 124.

\section{The WN8 Runaway WR 124 and Its Nebula}

The number of Galactic runaway WR stars is scarce, and only a handful of objects with high altitude over the Galactic plane are identified (see Rosslowe \& Crowther 2015). Among them is WR 124 (a.k.a. Merrill's star), the fastest runaway WR star in the Galaxy (see Table 1). Moffat et al. (1982) and Moffat \& Shara (1986) found that WR 124 shows photometric and spectral variability with a period of $\sim 2.4$ days. Moffat et al. (1982) argued that if the variability is due to the presence of a companion, its mass should be $\gtrsim 1 M_{\odot}$, i.e., in the NS mass range. Marchenko et al. (1998b) concluded that the radial velocity measurements of WR 124 could not be used as a reliable identification of orbital motion in a binary, mainly because of the quality of the existing data. Hipparcos light curves of WR 124 obtained between 1990 March 9 and 1993 March 6 do not show periodic variability.

WR 124 is surrounded by the distinct nebula M1-67 (Figure 1). This is a young nebula with a dynamical age of $\sim 10^{4}$ yr that has experienced very little mixing with the surrounding interstellar medium (e.g., Esteban et al. 1991; Fernández-Martín et al. 2013). Figure 1 presents a Hubble Space Telescope (HST) image of M1-67 in comparison with a composite mid-infrared (mid-IR) image from the Herschel, Spitzer, and WISE telescopes. The HST image shows in great detail the clumpy morphology of M1-67 (e.g., Marchenko et al. 2010). Although of lower resolution, the mid-IR image also shows a clumpy distribution of material. The remarkable feature traced by the Spitzer $24 \mu \mathrm{m}$ image is the bipolar nebular morphology. M1-67 exhibits two blowouts toward the northwest and southeast directions. This bipolar structure indicates an axisymmetric geometry of the system probably provoked by a companion (e.g., Chu \& Treffers 1981). Particularly notable is that when compared to other WR nebulae, M1-67 displays a near-complete absence of oxygen yet is highly nitrogen enriched, suggesting that most of the oxygen has been processed via the CNO cycle (Chu \& Treffers 1981; Esteban et al. 1991; Fernández-Martín et al. 2013).

The distance to WR 124 has been a matter of discussion. A geometric distance determination has been performed using HST observations of M1-67. Marchenko et al. (2010) used nebular images of two epochs separated by $11.26 \mathrm{yr}$ and determined a distance of $d=3.35 \mathrm{kpc}$. Recently, the Gaia Data Release 2 reported a parallax for WR 124 of $1.15 \times 10^{-4}$ arcsec (Gaia Collaboration et al. 2018), translating to a distance of $\sim 8.7 \pm$ $2.6 \mathrm{kpc}$, consistent with the $8.4 \mathrm{kpc}$ spectroscopic distance from Hamann et al. (2006).

\section{Observations and Results}

In an attempt to probe the presence of a NS deeply embedded in WR 124 wind, we obtained observations with the Chandra X-ray observatory. The observations were carried out on 2017 July 10-14 using the Advanced Camera for Imaging and Spectroscopy (ACIS)-S camera. The observations consist of four pointings with exposure times of $27.69 \mathrm{ks}, 24.64 \mathrm{ks}, 28.45 \mathrm{ks}$, and 13.48 ks (Obs. IDs: 18929, 20108, 20109, and 20110; PI: L. M. Oskinova). The total exposure time is $94.24 \mathrm{ks}$.

The data have been analyzed using the CIAO software package v. 4.9 with the CALDB version 4.7.4. We combined the four observations using the CIAO task reproject_obs. Figure 2 shows X-ray images of M1-67 in the $0.3-10 \mathrm{keV}$ energy range. The right panel shows a close-up view of the central region around WR 124.

No diffuse X-ray emission is detected in M1-67. This nebula consists of matter ejected by the star, and thus the mechanism of its formation is different from the WR nebulae around such WNE stars as WR 6, WR 7, WR 18, and WR 136 (see Toalá et al. 2017, and references therein), in which a fast-wind-slowwind interaction is thought to take place (e.g., Garcia-Segura \& Mac Low 1995). M1-67 around WR 124 is the third WR nebula around a WN8 star without any hint of diffuse X-ray emission, along with RCW 58 (WR 40) and the nebula around WR 16 (Gosset et al. 2005; Toalá \& Guerrero 2013).

The right panel in Figure 2 reveals some X-ray emission within $3^{\prime \prime}$ around WR 124 . There are only four net counts at the location of WR 124, all of which are detected at energies above $1 \mathrm{keV}$. This corresponds to an average count rate of $\sim 4 \times 10^{-5}$ counts $\mathrm{s}^{-1}$. Using Chandra PIMMS count rate simulator, we assumed a thermal plasma model typical for a WR star (Ignace et al. 2003). Corrected for the interstellar absorption, the X-ray flux is $10^{-15} \mathrm{erg} \mathrm{cm}^{-2} \mathrm{~s}^{-1}$ in the $0.2-10 \mathrm{keV}$ energy range. At a distance of $8.7 \mathrm{kpc}$ this corresponds to an X-ray luminosity of $L_{\mathrm{X}} \approx 10^{31} \mathrm{erg} \mathrm{s}^{-1}$. 

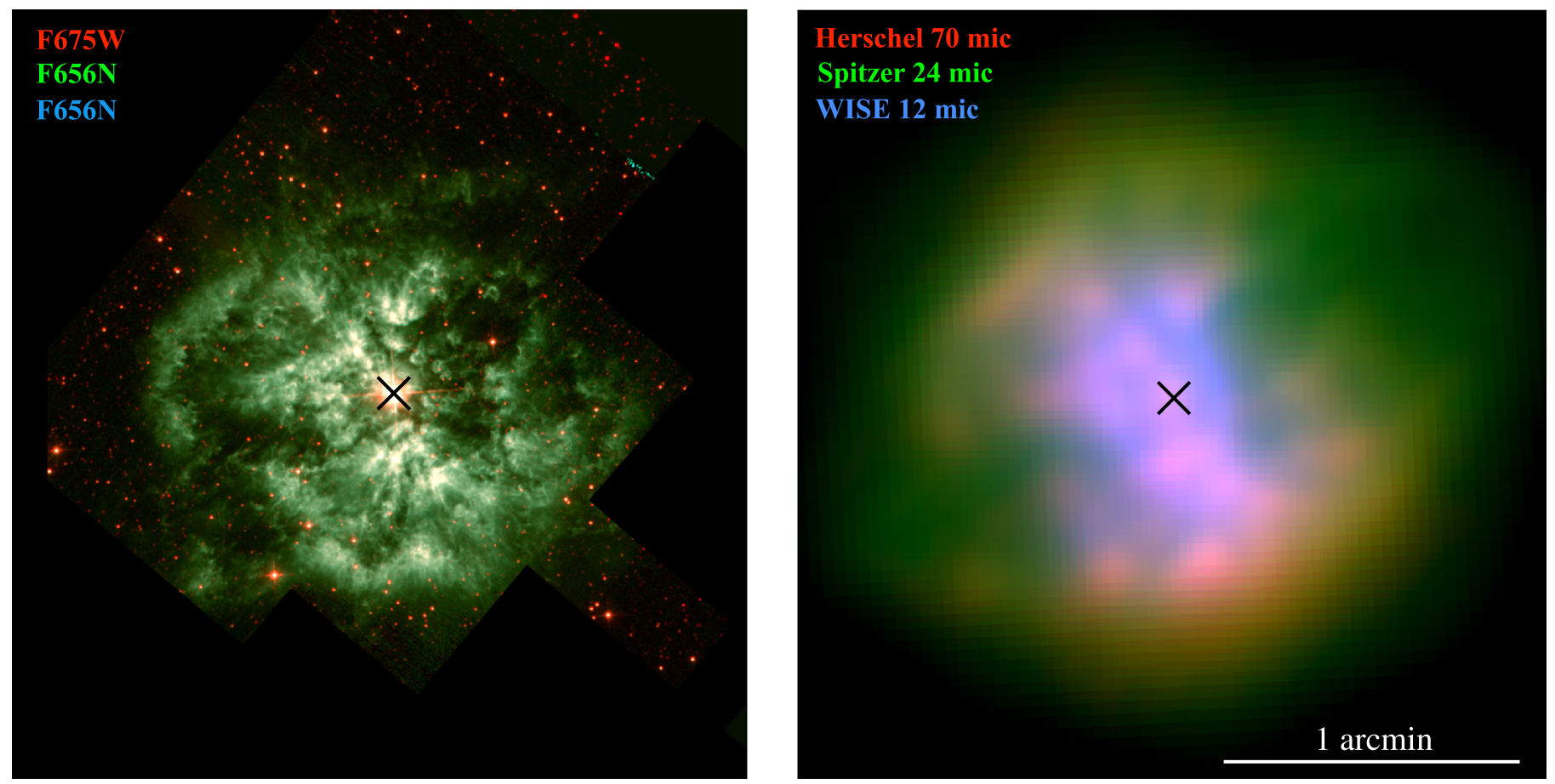

Figure 1. Color-composite pictures of M1-67. Left: HST image. Right: IR image. The position of WR 124 is shown with a cross in the two panels. Both images have the same field of view. North is up and east is to the left.

\section{Discussion}

The lack of diffuse X-ray emission from WR nebulae around WN8 stars suggests that their winds are not as efficient as those from WN4-6-type stars in producing hot bubbles, or most likely, that the formation scenario of WR nebulae around these stars is different from the fast-wind-slow-wind scenario. The morphologies of nebulae around WN8 stars, M1-67 (WR 124), RCW 58 (WR 40) and the nebula around WR 16, are similar. These WR nebulae exhibit a disrupted morphology (Marston et al. 1994; Gruendl et al. 2000; Marchenko et al. 2010) that might have caused the hot gas to leak out of the nebulae. The weak X-ray emission from the central source, WR 124, is consistent with the non-detection or marginal X-ray detections of other WN8-type stars (see Gosset et al. 2005; Skinner et al. 2012).

WR 124 is a runaway star surrounded by a young $\left(10^{4} \mathrm{yr}\right)$ enriched bipolar nebula, and is a faint but hard X-ray source. These strongly suggest that during its evolution WR 124 interacted with a binary companion. What could be the possible nature of this companion?

1) WR 124 could be a secondary star in a massive binary disrupted by the primary SN. As an order of magnitude estimate, using the star radial velocity of $2 \times 10^{-4} \mathrm{pc} \mathrm{yr}^{-1}$, and its height above the Galactic plane of $|z| \approx 500 \mathrm{pc}$, the SN took place $\lesssim 1$ Myr ago. Since then WR 124 has evolved as a single star, and ejected the M1-67 nebula during its previous evolutionary stage. The faint X-ray emission may be due to intrinsic shocks in the WR wind, but given the quite low wind velocity in WR 124, this seems unlikely. This scenario does not explain the bipolar shape of M1-67 nebula.

2) A massive early-type companion is ruled out. Such a companion would have signatures in the optical and $\mathrm{X}$-ray spectra. The colliding winds in $\mathrm{WR}+\mathrm{OB}$ binaries are strong X-ray sources (see Munoz et al. 2017). A massive late-type companion is equally unlikely, as it would have been noticed, for example, via IR and optical photometry and spectroscopy. An intermediate- or low-mass (e.g., solar-type) companion also seems unlikely. The formation timescale of these stars is $\sim 100 \mathrm{Myr}$, during which they are observed as active T Tauri type objects. There is no reason to suspect a $\mathrm{T}$ Tau type companion in WR 124.

3) Low-mass $\mathrm{He}$ ("stripped") companions in massive binaries are predicted by population syntheses. Because of their long lifetimes, they should be present in a significant fraction of massive binaries and HMXBs. The standard evolutionary scenarios predict that a stripped $\mathrm{He}$ star will collapse before the secondary evolves to the WR stage. However, the exact evolutionary path is decided by the race between evolutionary timescales of the stripped He-burning primary and the rejuvenated secondary, which gained considerable mass. Perhaps the hydrogen-poor WR 124 might be a fast-evolving mass gainer with a stripped companion. The stripped star should have quite a low mass to avoid driving a strong stellar wind, or we would detect X-rays from the colliding stellar winds. Under this scenario, no $\mathrm{SNe}$ occurred and the runaway velocity of WR 124 could be due to by dynamic interactions in a maternal massive star cluster (e.g., Oh \& Kroupa 2016), although a simple estimate of the kinematical timescale is large to support this idea.

4) An NS is buried within the WR star, that is, a TŹ scenario as suggested by Foellmi \& Moffat (2002). The problem with this suggestion is that TŻOs are expected to appear as red supergiants (Biehle 1991; Cannon 1993). There are no known hydrostatic solutions of stars with neutron cores that are hot enough to appear as a WN star. However, the formation phase of a TŻO is not yet fully explored (e.g., Biehle 1991; Podsiadlowski et al. 1995; Fryer et al. 1996). A merger of a blue supergiant with an NS may lead to a significant ejection of material (perhaps producing the M1-67 nebula), and might 

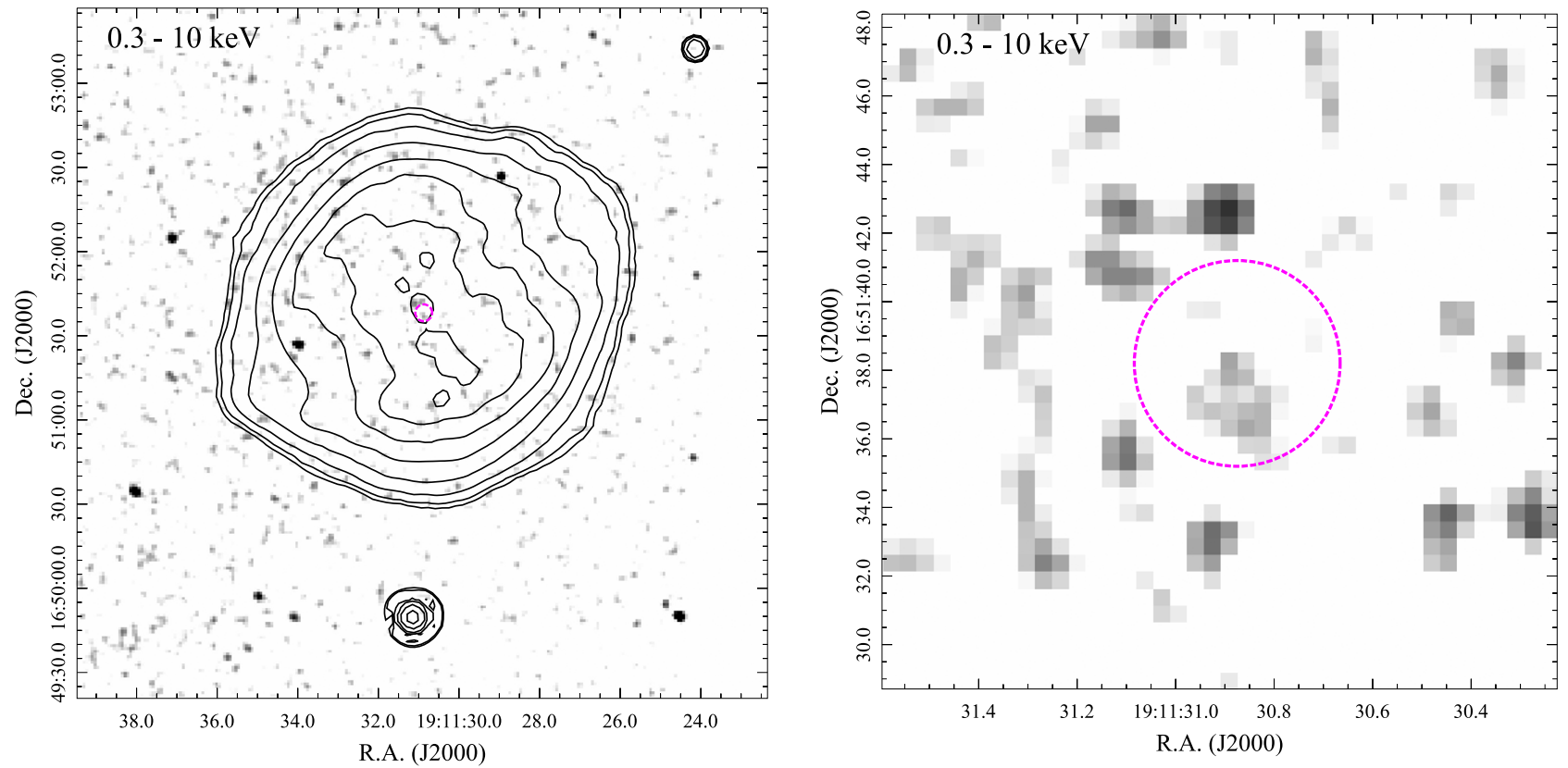

Figure 2. Left: Chandra ACIS-S X-ray image of the field of view around WR 124 in the $0.3-10 \mathrm{keV}$ energy range and combining all four observations. The contours show the $24 \mu \mathrm{m}$ emission as detected by Spitzer Multi-Band Imaging Photometer (see Figure 1). Right: the same as left panel but smaller field of view around WR 124. The magenta circle in both panels is centered at the position of WR 124 with an angular radius of $3^{\prime \prime}$.

temporarily look like a WN8 star (although there are no detailed calculations demonstrating this). This phase would be short-lived before the envelope has inflated to the red supergiant structure, which would occur on a thermal timescale (of the order of $10^{4} \mathrm{yr}$ and just compatible with the dynamical age of the nebula). Interestingly, Chevalier (2012) argued that the spiral-in of an NS or BH inside of a CE might be responsible for the ejected mass seen in SN IIn. However, this channel does not explain the abundance of the WN8 star and its nebula.

The end phase of a TŻO objects is also not well understood. The hydrostatic structure of TŻO models with massive envelopes is predicted to break down when either the envelope falls below a critical mass or the envelope runs out of $\mathrm{CNO}$ elements (Podsiadlowski et al. 1995). This is expected to lead to runaway accretion onto the NS and the possible formation of a BH. It is not known what such an object will look like initially (after the red supergiant envelope has collapsed) and whether there is any mass ejection connected with this collapse phase. But considering the uncertainties of this process, one cannot rule out that it could look like a WN star. This phase would also be relatively short-lived (on the thermal timescale of the envelope $\sim 10^{4} \mathrm{yr}$ ). Given the large uncertainties, a final stage of TŻO evolution might look like a WN8h object.

5) An NS companion exists on a close orbit around WR 124, similar to a persistent HMXBs (see Martínez-Núñez et al. 2017). To predict the X-ray luminosity of an NS embedded in a WN8 wind, we assume Bondi-Hoyle-type wind accretion. WR 124 was analyzed by means of modern non-local thermodynamic equilibrium stellar atmosphere model PoWR (Hamann et al. 2006). For the purpose of estimation, we adopt a generic WN8 stellar wind model from the WR model grid. ${ }^{9}$ The model provides the ionization structure, density, and velocity stratification in the stellar wind. With this information

\footnotetext{
9 www.astro.physik.uni-potsdam.de/ wrh/PoWR/powrgrid1.php
}

we compute the accretion luminosity of an NS located at different orbital separation using the formalism that accounts for the orbital velocities (Oskinova et al. 2012). We find that the accretion rate onto an NS $\left(m_{\mathrm{NS}}=1.4 M_{\odot}\right)$ is superEddington for orbital separations up to $8 R_{\star}$. In a system accreting at such high rates, the high X-ray flux and strong outflows are likely to disrupt the stellar wind. However, more realistic accretion models that account for magnetic gating and propeller mechanism (Bozzo et al. 2016) show that for orbital separations $a_{\mathrm{NS}}>1.5 R_{\star}$ much lower intrinsic X-ray luminosity $\left(<10^{36} \mathrm{erg} \mathrm{s}^{-1}\right)$ and ionization parameter are expected.

We used the PoWR model to compute the WN8 wind opacity for X-rays. The wind is optically thick to X-ray radiation in the Chandra passband up to $\sim 100 R_{\star}$. This easily explains the very low observed X-ray luminosity of WR 124$\mathrm{X}$-rays are fully absorbed in the stellar wind. Figure 3 shows the optical depth between an NS and an observer for different orbital separations. The WN8 star wind is extremely optically thick to X-rays. For example, at $a_{\mathrm{NS}}=2 R_{\star}$ the optical depth at $\lambda=10 \AA$ is $\tau_{\lambda} \approx 100$, while $\tau_{\lambda} \approx 50$ for an NS located at $8 R_{\star}$. This means that X-ray emission produced by an accreting NS embedded in the stellar wind is completely absorbed.

The high stellar wind opacity for the X-rays is mainly due to the K-shell absorption by N III-N V. The plot shown in Figure 3 does not include the effect of X-ray photoionization, which would reduce the wind opacity in the vicinity of the accreting source. While at the X-ray luminosities typical for accreting NSs, the size of the photoionized region is significantly smaller that $100 R_{*}$, the situation may be different for accreting BHs and their high X-ray luminosities.

The model calculations show that a marginal detection of X-rays from WR 124 does not rule out the presence of an NS embedded in its wind. In this case, the M1-67 nebula might be a remnant of a $\mathrm{CE}$ occurring from a previous evolutionary phase (e.g., an LBV). 


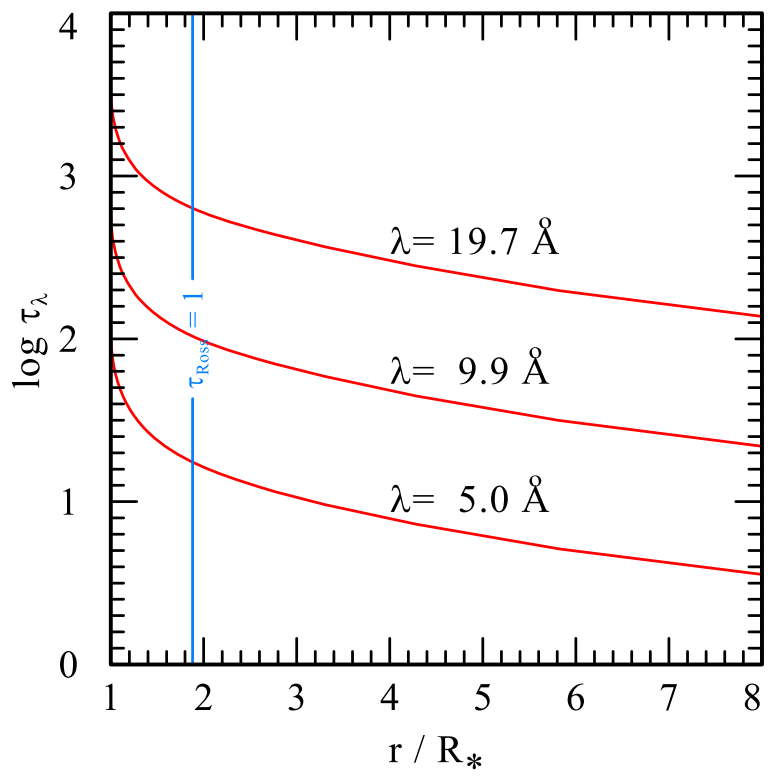

Figure 3. Model WN8 wind optical depth at different wavelengths as a function of the distance from the stellar center. The thin blue line shows the photospheric radius defined by a Rosseland optical depth of unity.

\section{Conclusions}

We present deep $\sim 100 \mathrm{ks}$ Chandra X-ray observations of one of the most promising candidate $\mathrm{WR}+\mathrm{NS}$ binaries in our Galaxy, the WN8-type star WR 124. We detect the star with $L_{\mathrm{X}} \lesssim 10^{31} \mathrm{erg} \mathrm{s}^{-1}$, which is similar to other WN8-type stars.

Different evolutionary scenarios on the formation of WR 124 and its nebula are considered. We reason that WR 124 could be either an advanced evolutionary stage of a TŻO or, most likely, a binary hosting an NS.

We show that winds of WN8-type stars are significantly opaque to X-rays that could be produced by an accreting NS at orbital separations up to tens of stellar radii.

We conclude that the lack of strong X-rays from WR 124 does not rule out the presence of an accreting NS. The dense and opaque winds of WR stars effectively hide embedded NSs from X-ray detection.

The authors are indebted to the anonymous referee for a detailed revision of the manuscript and constructive suggestions. Support for this Letter was provided by the National Aeronautics and Space Administration through Chandra award No. G07-18014X issued by the Chandra X-ray Center, which is operated by the Smithsonian Astrophysical Observatory for and on behalf of the NASA contract NAS8-03060. J.A.T., M.A.G., and H.T. are funded by UNAM DGAPA PAPIIT project IA100318. L.M.O. acknowledges support by the DLR grant 50 OR 1508 and partial support by the Russian Government Program of Competitive Growth of Kazan Federal University. A.A.C.S. is supported by the Deutsche Forschungsgemeinschaft (DFG) under grant HA $1455 / 26$ and would like to thank STFC for funding under grant No. ST/R000565/1. Y.-H.C. acknowledges support from the Ministry of Science and Technology of Taiwan. T.S. acknowledges support from the German Verbundforschung (DLR) grant 50 OR 1612. J.M.T. acknowledges support from ESP2017-85691-P.

\section{ORCID iDs}

J. A. Toalá (杜宇君) (10 https://orcid.org/0000-0002-5406-0813

R. Ignace (i) https://orcid.org/0000-0002-7204-5502

T. Shenar (i) https://orcid.org/0000-0003-0642-8107

M. A. Guerrero (iD https://orcid.org/0000-0002-7759-106X

J. M. Torrejón (iD https://orcid.org/0000-0002-5967-5163

\section{References}

Barniske, A., Oskinova, L. M., \& Hamann, W.-R. 2008, A\&A, 486, 971 Biehle, G. T. 1991, ApJ, 380, 167

Bozzo, E., Oskinova, L., Feldmeier, A., \& Falanga, M. 2016, A\&A, 589, 102 Cannon, R. C. 1993, MNRAS, 263, 817

Chené, A.-N., Foellmi, C., Marchenko, S. V., et al. 2011, A\&A, 530, A151

Chevalier, R. A. 2012, ApJL, 752, L2

Chu, Y.-H., \& Treffers, R. R. 1981, ApJ, 249, 586

De Donder, E., Vanbeveren, D., \& van Bever, J. 1997, A\&A, 318, 812

Esteban, C., Vilchez, J. M., Manchado, A., \& Smith, L. J. 1991, A\&A, 244, 205

Fernández-Martín, A., Vílchez, J. M., Pérez-Montero, E., et al. 2013, A\&A, 554, A104

Foellmi, C., \& Moffat, A. F. J. 2002, in ASP Conf. Proc. 263, Stellar Collisions, Mergers and their Consequences, ed. M. M. Shara (San Francisco, CA: ASP), 123

Fryer, C. L., Benz, W., \& Herant, M. 1996, ApJ, 460, 801

Gaia Collaboration, Brown, A. G. A., Vallenari, A., et al. 2018, arXiv:1804. 09365

Garcia-Segura, G., \& Mac Low, M.-M. 1995, ApJ, 455, 145

Gosset, E., Nazé, Y., Claeskens, J.-F., et al. 2005, A\&A, 429, 685

Gruendl, R. A., Chu, Y.-H., Dunne, B. C., \& Points, S. D. 2000, AJ, 120, 2670

Hamann, W.-R., Gräfener, G., \& Liermann, A. 2006, A\&A, 457, 1015

Ignace, R., Oskinova, L. M., \& Brown, J. C. 2003, A\&A, 408, 353

Jiang, Y.-F., Cantiello, M., Bildsten, L., et al. 2018, Natur, 561, 498

Kharchenko, Scholz, R.-D., Piskunov, A. E., et al. 2007, AN, 328, 889

Koljonen, K. I. I., Maccarone, T., McCollough, M. L., et al. 2018, A\&A, 612, A27

Lommen, D., Yungelson, L., van den Heuvel, E., et al. 2005, A\&A, 443, 231

MacLeod, M., \& Ramirez-Ruiz, E. 2015, ApJ, 803, 41

Marchenko, S. V., Moffat, A. F. J., \& Crowther, P. A. 2010, ApJL, 724, L90

Marchenko, S. V., Moffat, A. F. J., Eversberg, T., et al. 1998a, MNRAS, 294, 642

Marchenko, S. V., Moffat, A. F. J., van der Hucht, K. A., et al. 1998b, A\&A 331,1022

Marston, A. P., Yocum, D. R., Garcia-Segura, G., \& Chu, Y.-H. 1994, ApJS, 95,151

Martínez-Núñez, Kretschmar, P., Bozzo, E., et al. 2017, SSRv, 212, 59

Moffat, A. F. J. 1989, ApJ, 347, 373

Moffat, A. F. J., Lamontagne, R., \& Seggewiss, W. 1982, A\&A, 114, 135

Moffat, A. F. J., Marchenko, S. V., Seggewiss, W., et al. 1998, A\&A, 331, 949

Moffat, A. F. J., \& Shara, M. M. 1986, AJ, 92, 952

Munoz, M., Moffat, A. F. J., Hill, G. M., et al. 2017, MNRAS, 467, 3105

Oh, S., \& Kroupa, P. 2016, A\&A, 590, 107

Oskinova, L. M., Feldmeier, A., \& Kretschmar, P. 2012, MNRAS, 421, 2820

Oskinova, L. M., Ignace, R., Hamann, W.-R., Pollock, A. M. T., \& Brown, J. C. 2003, A\&A, 402, 755

Paczynski, B. 1967, AcA, 17, 355

Podsiadlowski, P., Cannon, R. C., \& Rees, M. J. 1995, MNRAS, 274, 485

Postnov, K. A., \& Yungelson, L. R. 2014, LRR, 17, 3, 166

Rosslowe, C. K., \& Crowther, P. A. 2015, MNRAS, 447, 2322

Sander, A., Hamann, W.-R., Todt, H., et al. 2018, arXiv:180704293

Shenar, T., Hainich, R., Todt, H., et al. 2016, A\&A, 591, 22

Skinner, S. L., Zhekov, S. A., Güdel, M., Schmutz, W., \& Sokal, K. R. 2012, AJ, 143, 116

Terman, J. L., Taam, R. E., \& Hernquist, L. 1995, ApJ, 445, 367

Toalá, J. A., \& Guerrero, M. A. 2013, A\&A, 559, A52

Toalá, J. A., Marston, A. P., Guerrero, M. A., Chu, Y.-H., \& Gruendl, R. A. 2017, ApJ, 846, 76

van den Heuvel, E. P. J., Portegies Zwart, S. F., \& de Mink, S. E. 2017, MNRAS, 471, 4256

van Kerkwijk, M. H., Charles, P. A., Geballe, T. R., et al. 1992, Natur, 355, 703

Vanbeveren, D., De Loore, C., \& Van Rensbergen, W. 1998, A\&ARv, 9, 63

Woosley, S. E., \& Bloom, J. S. 2006, ARA\&A, 44, 507 